\title{
Ion Association and Electrolyte Structure at Surface Films in Lithium-ion Batteries
}

Justin R. Pinca, William G. Duborg, Ryan Jorn*

Department of Chemistry, Villanova University, Villanova, Pennsylvania 19085, United States

\section{Supporting Information}

Table S1. Compilation of the number of molecules used in each SEI simulation.

\begin{tabular}{lccccc}
\hline \multicolumn{1}{c}{ SEI Composition } & $\mathbf{E C}$ & $\mathbf{L i P F}_{\mathbf{6}}$ & $\mathbf{L i}_{2} \mathbf{E D C}$ & $\mathbf{L i F}$ & $\mathbf{L i}_{2} \mathbf{C O}_{3}$ \\
\hline \hline $\mathrm{Li}_{2}$ EDC (non-scaled) & 4158 & 432 & 909 & - & - \\
$\mathrm{Li}_{2} \mathrm{EDC}$ (q-scaled) & 4023 & 419 & 909 & - & - \\
$\mathrm{LiF}$ (non-scaled) & 6044 & 632 & - & 12,960 & - \\
$\mathrm{LiF}$ (q-scaled) & 5852 & 612 & - & 12,960 & - \\
$\mathrm{Li}_{2} \mathrm{CO}_{3}$ (non-scaled) & 6076 & 632 & - & - & 4023 \\
$\mathrm{Li}_{2} \mathrm{CO}_{3}$ (q-scaled) & 5884 & 612 & - & - & 4023
\end{tabular}

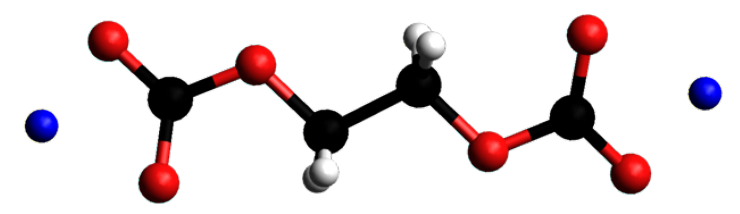

Figure S1. Molecular structure for $\mathrm{Li}_{2} \mathrm{EDC}$ (for color coding of atoms see Figure 1). 


\section{a. Comparing Bulk Electrolyte Non-scaled and Q-scaled Models}

The following figures provide additional structural information about the bulk electrolyte. Figure S2-S3 provide structural information for ion solvation at 313K, while Figures S4-S5 show results at the elevated temperature used for the interface simulations discussed in the manuscript.

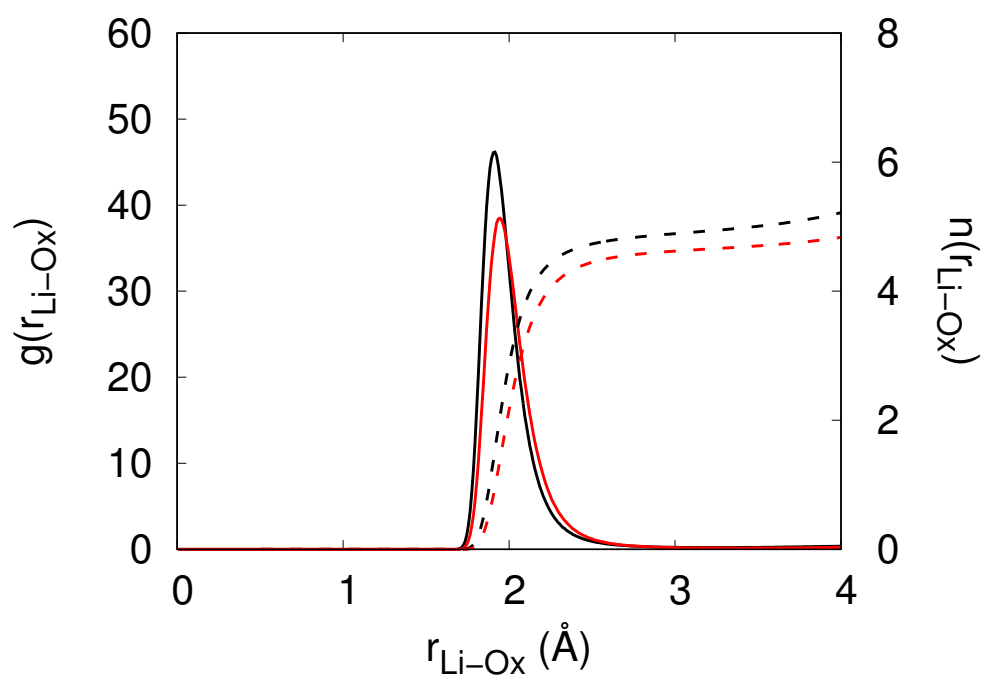

Figure S2. The radial distribution functions (solid lines) are shown for the lithium-EC carbonyl oxygen pair in the bulk electrolyte at $313 \mathrm{~K}$ described by the non-scaled (black) and q-scaled (red) force fields. The coordination number for oxygens surrounding lithium ions are also shown for both models (dashed lines).

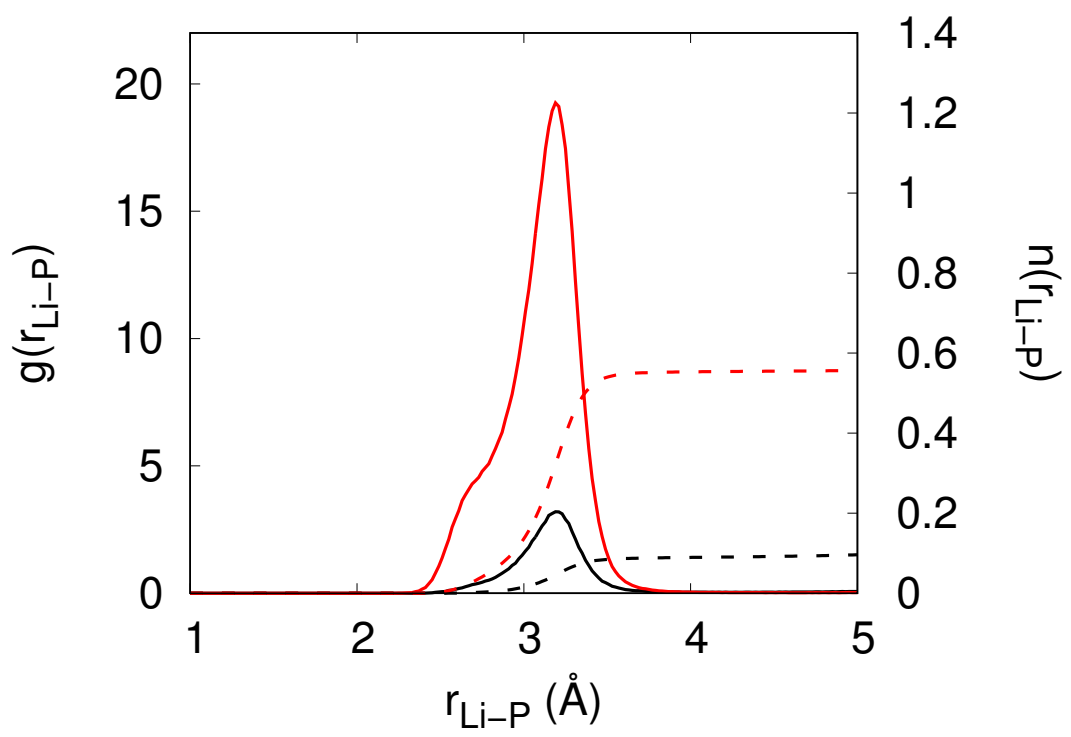

Figure S3. The radial distribution functions (solid lines) are shown for the lithium-phosphorous pair in the bulk electrolyte at $313 \mathrm{~K}$ described by the non-scaled (black) and q-scaled (red) force fields. The coordination number for phosphorous atoms surrounding the lithium ions are also shown for both models (dashed lines). 


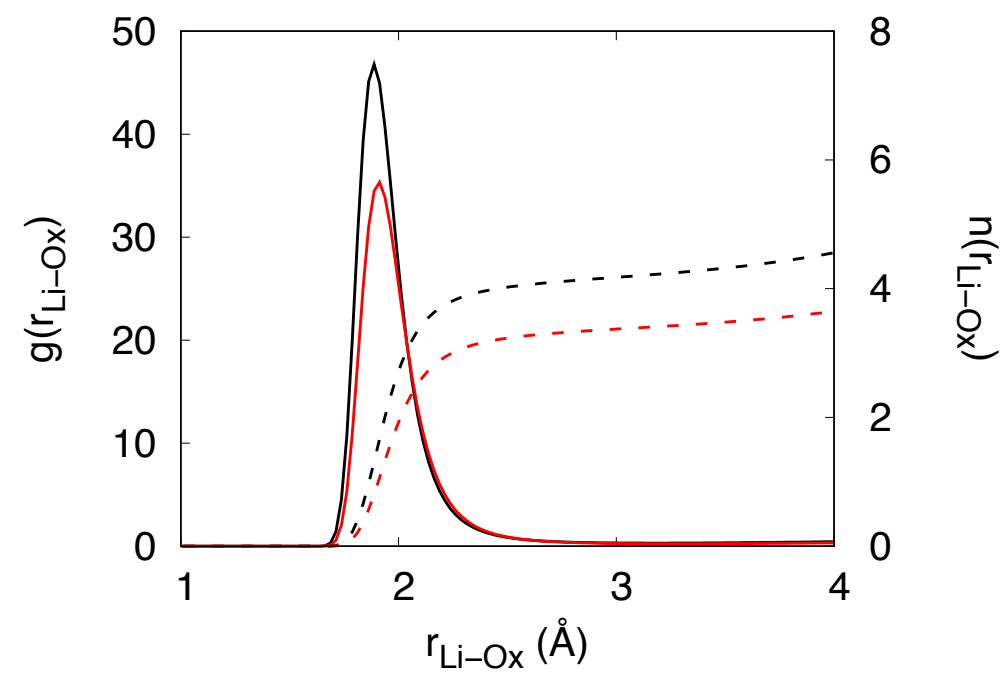

Figure S4. The radial distribution functions (solid lines) are shown for the lithium-EC carbonyl oxygen pair in the bulk electrolyte at $453 \mathrm{~K}$ described by the non-scaled (black) and q-scaled (red) force fields. The coordination number for oxygens surrounding lithium ions are also shown for both models (dashed lines).

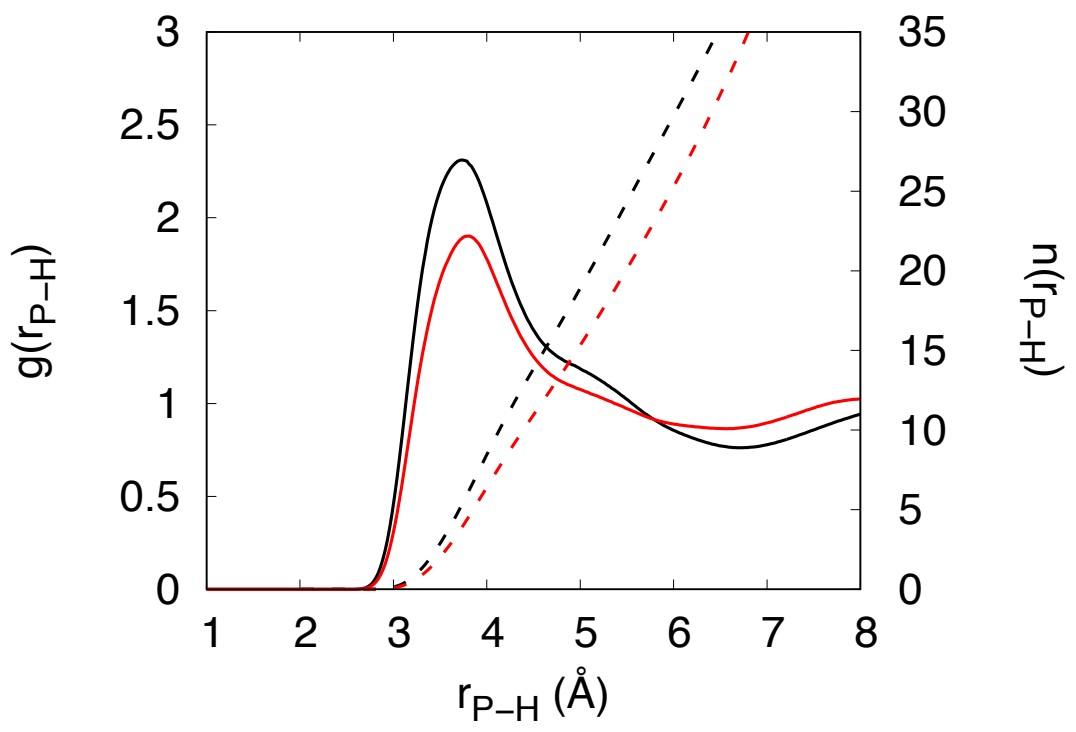

Figure S5. The radial distribution functions (solid lines) are shown for the phosphorous-EC hydrogen pair in the bulk electrolyte at $453 \mathrm{~K}$ described by the non-scaled (black) and q-scaled (red) force fields. The coordination number for hydrogens surrounding the phosphorous are also shown for both models (dashed lines). 


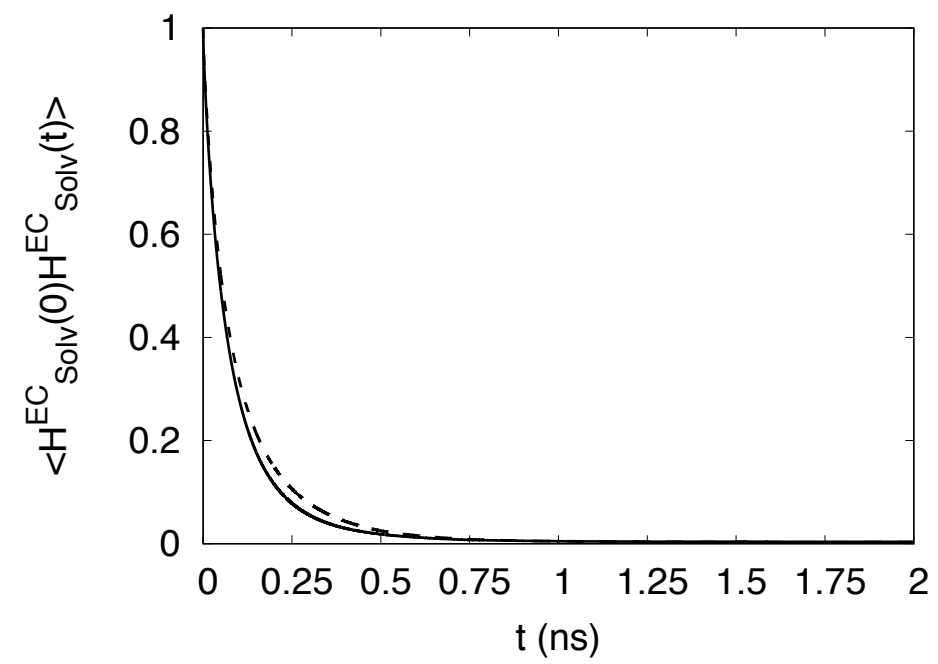

Figure S6. The solvation residence correlation function is shown for EC coordinated to $\mathrm{Li}^{+}$in both the non-scaled(solid) and q-scaled(dashed) model at $453 \mathrm{~K}$.

\section{b. Ion Accumulation at SEI Interfaces}

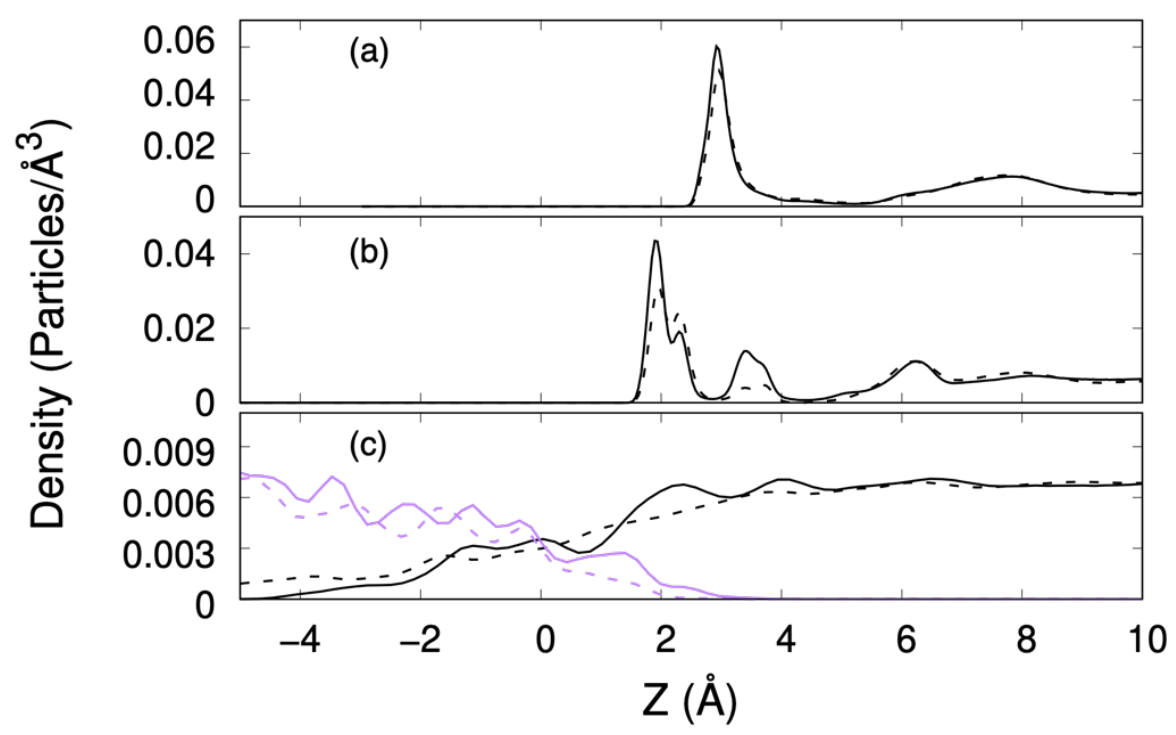

Figure S7. Plots of the EC (black) center-of-mass density at the SEI interface using the non-scaled (solid) and q-scaled (dashed) force fields. Results are shown at the (a) $\mathrm{LiF}[100]$, (b) $\mathrm{Li}_{2} \mathrm{CO}_{3}[010]$, and (c) amorphous $\mathrm{Li}_{2} \mathrm{EDC}$ surfaces and the origin is set to the location of the surface film. In the case of the $\mathrm{Li}_{2} \mathrm{EDC}$ surface, the center-of-mass density for the $\mathrm{Li}_{2} \mathrm{EDC}$ (purple) is also shown. 


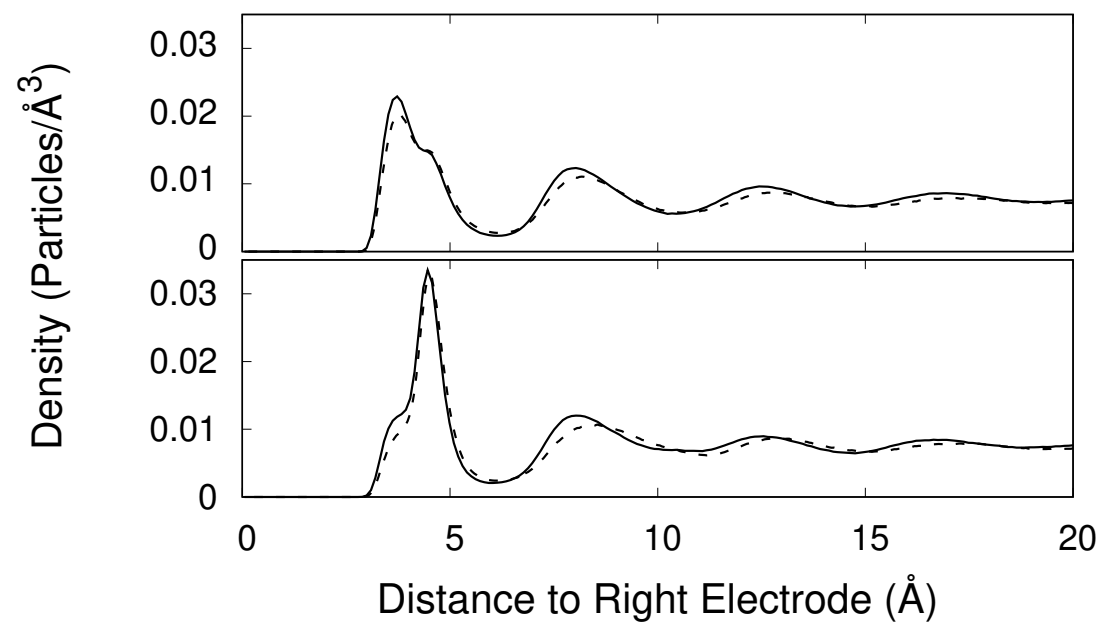

Figure S8. Plots of the EC center-of-mass density at the graphite edge surface of the right electrode at $0 \mathrm{~V}$ (top panel) and at a total potential drop of 3V (bottom panel) across the simulation cell. Results are shown for the non-scaled model (solid) and q-scaled model (dashed).

\section{c. Ion Association at SEI Interfaces}

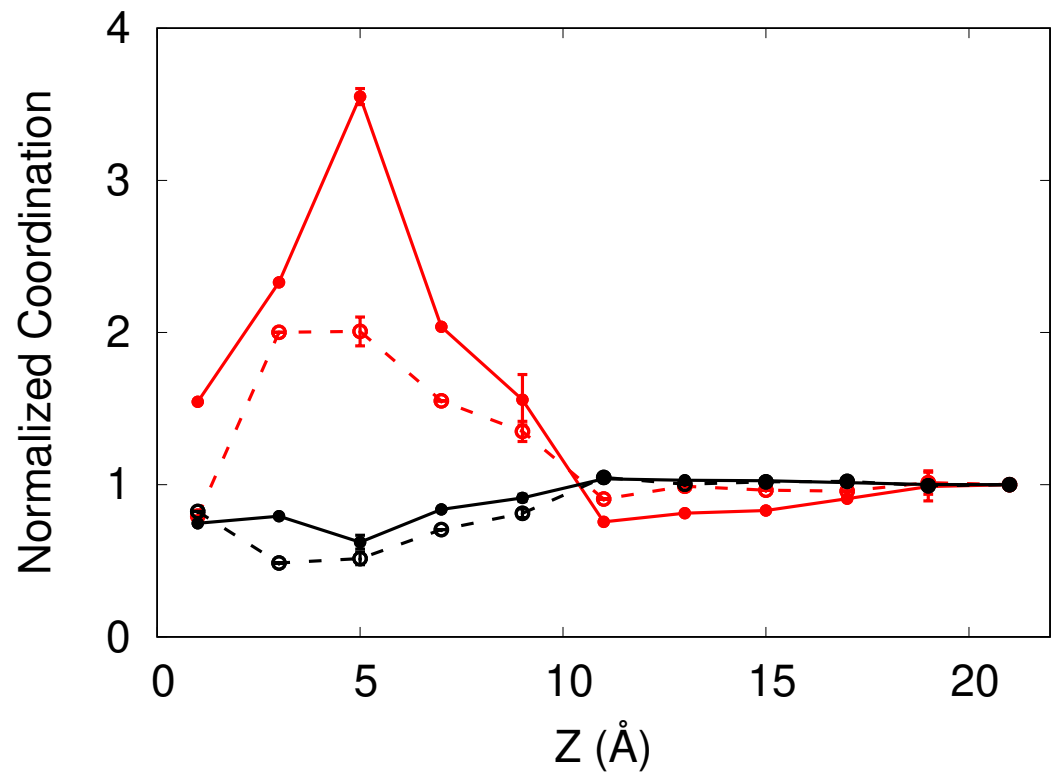

Figure S9. Coordination of lithium ions by $\mathrm{PF}_{6}{ }^{-}$(red) and EC (black) as a function of location with respect to the LiF surface. All curves are normalized to their bulk values and the results from the non-scaled (solid) and q-scaled (dashed) force fields are compared. 


\section{d. Electrolyte Dynamics at the Interface}

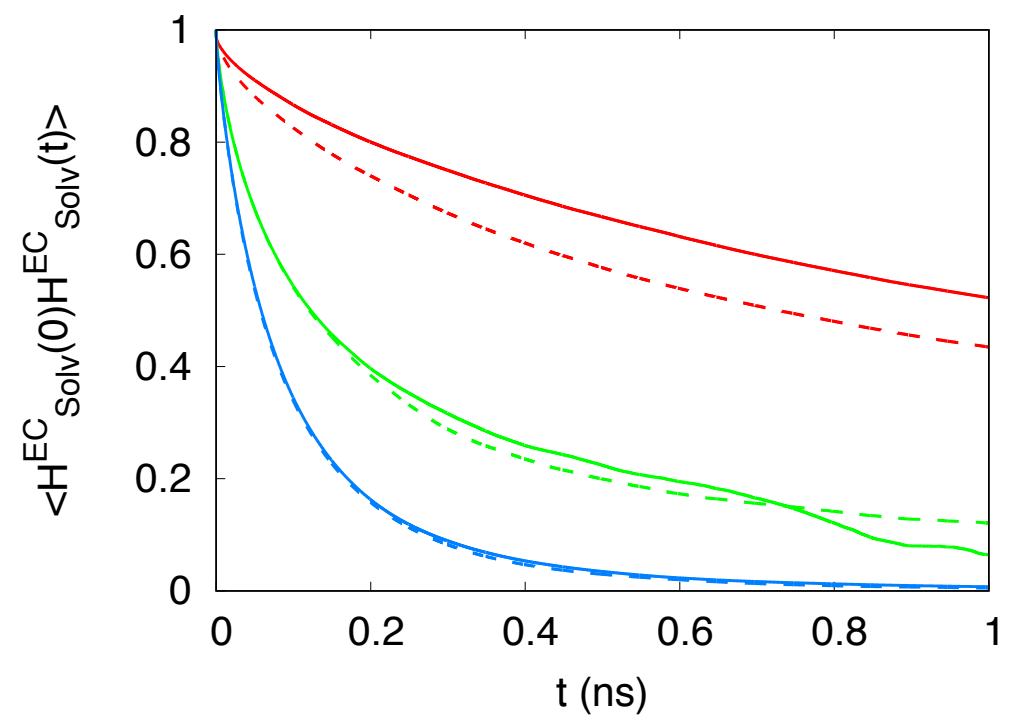

Figure S10. The solvation correlation function for EC coordinating lithium ions is shown for 5$7 \AA ̊$ layers centered around $3.5 \AA$ from the $\mathrm{Li}_{2}$ EDC surface (red), $7.5 \AA$ from the surface (green), and in the middle of the simulation cell (blue). Results are shown using the non-scaled (solid) and q-scaled (dashed) models.

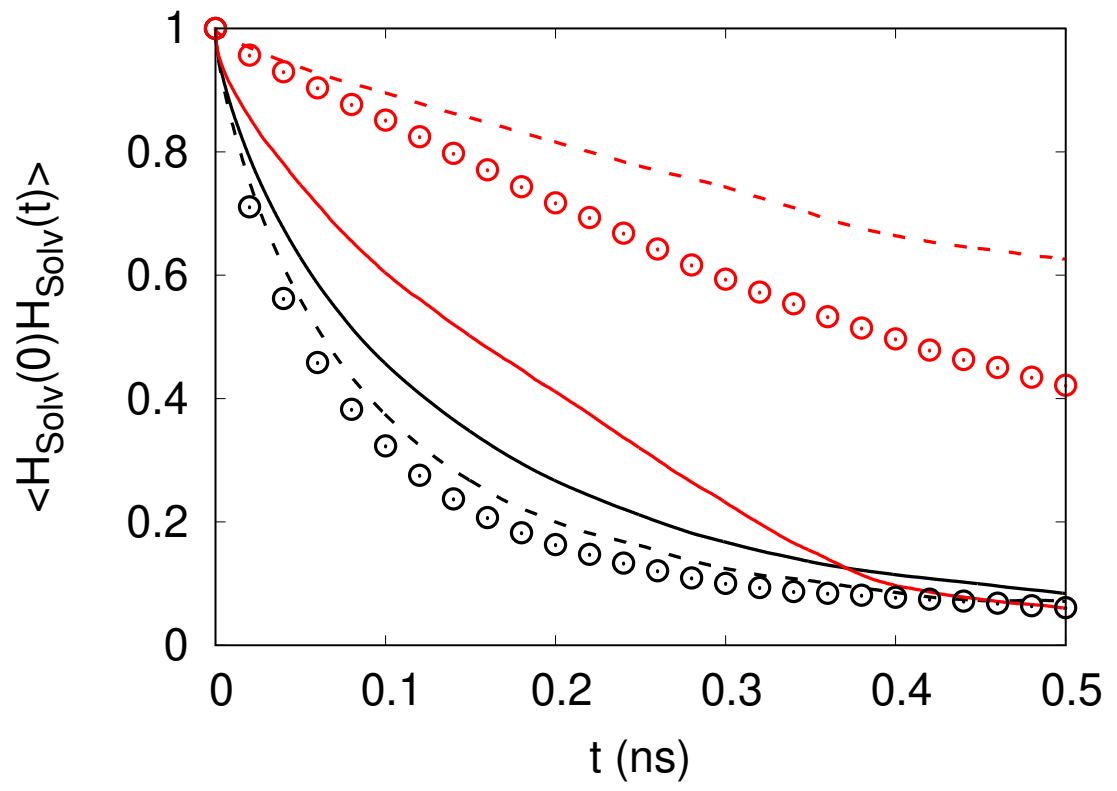

Figure S11. Solvent correlation functions shown for EC (black) and $\mathrm{PF}_{6}{ }^{-}$(red) coordinated to lithium ions in the first $12 \AA$ from the right graphite electrode using the non-scaled (solid) and qscaled (dashed) models for EC. Also shown are the results from the $3 \mathrm{~V}$ simulation using the qscaled model (circles). 